ROM2F/2004-04

MS-TP-04-02

DESY 04-022

\title{
The continuum limit of the quark mass step scaling function in quenched lattice QCD
}

\author{
$\bar{\nexists}_{\text {Colubersion }}$ \\ M. Guagnelli ${ }^{a}$, J. Heitger ${ }^{b}$, F. Palombi ${ }^{c, a}$, C. Pena ${ }^{d}$ and A. Vladikas ${ }^{a}$ \\ ${ }^{a}$ INFN, Sezione di Roma II \\ c/o Dipartimento di Fisica, Università di Roma "Tor Vergata" \\ Via della Ricerca Scientifica 1, I-00133 Rome, Italy \\ ${ }^{b}$ Westfälische Wilhelms-Universität Münster, Institut für Theoretische Physik \\ Wilhelm-Klemm-Strasse 9, D-48149 Münster, Germany \\ c "E. Fermi" Research Center \\ c/o Compendio Viminale, pal. F, I-00184 Rome, Italy \\ d DESY, Theory Group \\ Notkestrasse 85, D-22607 Hamburg, Germany
}

\begin{abstract}
The renormalisation group running of the quark mass is determined non-perturbatively for a large range of scales, by computing the step scaling function in the Schrödinger Functional formalism of quenched lattice QCD both with and without $\mathrm{O}(a)$ improvement. A one-loop perturbative calculation of the discretisation effects has been carried out for both the Wilson and the Clover-improved actions and for a large number of lattice resolutions. The non-perturbative computation yields continuum results which are regularisation independent, thus providing convincing evidence for the uniqueness of the continuum limit. As a byproduct, the ratio of the renormalisation group invariant quark mass to the quark mass, renormalised at a hadronic scale, is obtained with very high accuracy.
\end{abstract}




\section{Introduction}

The renormalisation group running of the QCD fundamental parameters, namely the renormalised gauge coupling and quark masses, has now been computed nonperturbatively for a large range of scales, albeit in the limit of infinitely heavy sea quarks; see refs. 11, 2]. These results have been obtained using lattice regularised quenched QCD with Wilson fermions, prior to extrapolating to the continuum limit. In the case of the quark mass running, Symanzik improvement was an important element in reducing the extrapolation uncertainties, as it implies that the dominant systematic effects due to the finiteness of the UV cutoff $a^{-1}$ are $\mathrm{O}\left(a^{2}\right)$. The case of QCD with two dynamical flavours has been investigated in 3 .

The continuum limit of lattice QCD is known to exist to all orders of perturbation theory (PT) 4. Beyond PT this issue has been addressed by numerical simulation. The strategy consists in implementing different regularisations which formally correspond to the same Field Theory (QCD in our case) and in establishing the universality of the continuum limit of given renormalised physical quantities, computed with different regulators. This sometimes turns out to be less straightforward than expected; e.g. see the discussion on the universality of the continuum limit of spin and sigma models in refs. 5. In pure $\mathrm{SU}(2)$ gauge theory, universality has been tested by computing two different non-perturbatively defined running couplings over a large range of energies 6]. In pure $\mathrm{SU}(3)$ gauge theory, evidence of universality has been recently found in a study of the scaling properties of the deconfining temperature with different gauge actions [7].

In the present work we extend these ideas to the step scaling function (SSF) of the quark mass in quenched QCD. This quantity has been calculated in ref. 2] from an improved action; here it is also evaluated from an unimproved action. The continuum SSF, computed for a large range of renormalisation scales, is found to be independent of these regularisation details, providing evidence for a universal continuum limit. In this respect our study parallels closely the one of ref. [8, which was dealing with the SSF of the operator corresponding to the average momentum of non-singlet parton densities. In comparison, our quantity is particularly simple, as we essentially compute ratios of two-point functions. This allows us to have an excellent control of both statistical and systematic errors. As a byproduct we recalculate the flavour independent ratio of the renormalisation group invariant (RGI) quark mass to the renormalised one (at a given hadronic scale). We obtain a result compatible to the original one of ref. 2] but fairly more accurate.

A study of discretisation effects in the SSF has also been performed, for both Wilson and Clover actions, in one-loop PT. This calculation has been carried out for a large number of lattice resolutions. We find that lowest order perturbation theory greatly underestimates the discretisation effects of the SSF. 


\section{The Schrödinger Functional and $\mathrm{O}(a)$ improvement}

In this section we gather the most relevant definitions and outline the properties of the quantities we are interested in. Most details are omitted, as they have been presented in previous works, which we will frequently refer to.

We adopt the lattice Schrödinger functional (SF) formalism [9, 10, 11]; more specifically we regularise QCD on a lattice of extension $L^{3} \times T$ (here $T=L$ always) with periodic boundary conditions in the space directions (up to a phase $\theta$ for the fermion fields) and Dirichlet boundary conditions in the Euclidean time direction [10, 11]. Otherwise the lattice gauge and fermionic field actions are of the standard Wilson type; their $\mathrm{O}(a)$ improved version is discussed below. The bare gauge coupling and quark mass are denoted by $g_{0}$ (with $\beta \equiv 6 / g_{0}^{2}$ ) and $m_{0}$ (with $\left.2 \kappa \equiv\left[a m_{0}+4\right]^{-1}\right)$, respectively. As we will be working in the quenched approximation, the bare gauge coupling $g_{0}$ and chiral point $\kappa_{\mathrm{c}}$ are functions of the lattice spacing $a$ alone. The chiral point is the value of the hopping parameter $\kappa$ for which the "current" quark mass, defined below, vanishes. The bare subtracted quark mass is defined as $a m_{\mathrm{q}}=\left[1 / \kappa-1 / \kappa_{\mathrm{c}}\right] / 2$, whereas an unrenormalised "current" quark mass is given by

$$
m\left(g_{0}\right)=\frac{\frac{1}{2}\left(\partial_{0}^{*}+\partial_{0}\right) f_{\mathrm{A}}\left(x_{0}\right)}{2 f_{\mathrm{P}}\left(x_{0}\right)},
$$

with $f_{\mathrm{X}}(\mathrm{X}=\mathrm{A}, \mathrm{P})$ the correlation functions of local bilinear operators

$$
f_{\mathrm{X}}\left(x_{0}\right)=-\frac{a^{6}}{2} \sum_{\mathbf{y}, \mathbf{z}}\left\langle\bar{\zeta}_{j}(\mathbf{y}) \gamma_{5} \zeta_{i}(\mathbf{z}) \bar{\psi}_{i}(x) \gamma_{\mathbf{x}} \psi_{j}(x)\right\rangle .
$$

The field indices $i, j$ label two distinct flavours; the "boundary fields" $\zeta$ are defined in ref. [12. For $\mathrm{X}=\mathrm{A}$ we have $\gamma_{\mathrm{X}}=\gamma_{0} \gamma_{5}$ and for $\mathrm{X}=\mathrm{P}$ we have $\gamma_{\mathrm{X}}=\gamma_{5}$. The forward and backward lattice time derivatives are denoted by $\partial_{0}$ and $\partial_{0}^{*}$ respectively ${ }^{1}$. We also define the correlation function of boundary fields

$$
f_{1}=-\frac{a^{12}}{2 L^{6}} \sum_{\mathbf{y}, \mathbf{z}, \mathbf{y}^{\prime}, \mathbf{z}^{\prime}}\left\langle\bar{\zeta}_{i}^{\prime}\left(\mathbf{y}^{\prime}\right) \gamma_{5} \zeta_{j}^{\prime}\left(\mathbf{z}^{\prime}\right) \bar{\zeta}_{j}(\mathbf{y}) \gamma_{5} \zeta_{i}(\mathbf{z})\right\rangle .
$$

Unprimed quantities are defined on the the $x_{0}=0$ boundary, primed ones on the $x_{0}=T$ one.

The $\mathrm{O}(a)$ Symanzik improvement of the above construction has been worked out in refs. 10, 12]. For the pure gauge action, it amounts to modifying it by introducing time-boundary counterterms proportional to $\left[c_{\mathrm{t}}\left(g_{0}^{2}\right)-1\right]$. For the fermionic action we must introduce the well-known clover counterterm in the lattice bulk, proportional to $c_{\mathrm{sw}}\left(g_{0}^{2}\right)$, and time-boundary counterterms proportional to $\left[\tilde{c}_{\mathrm{t}}\left(g_{0}^{2}\right)-1\right]$. Correlation

\footnotetext{
${ }^{1}$ We follow closely the notation of [2] 12], whither we refer for details.
} 
functions of composite operators such as eq. (2.2) may then also be $\mathrm{O}(a)$ improved by including in the lattice definition of these operators the appropriate counterterms. In the chiral limit there are no such counterterms for the pseudoscalar density $P(x)$, while the axial current $A_{0}(x)$ requires the addition of $\partial_{0} P$ with a coefficient $c_{\mathrm{A}}\left(g_{0}^{2}\right)$. The axial current is used in the computation of the bare quark mass, but, being scale independent, it is clearly not needed in the computation of its renormalisation group running. Thus $c_{\mathrm{A}}$ will play no rôle in the present work.

All these improvement coefficients may in principle be computed non-perturbatively for a range of values of the bare coupling $g_{0}$; for $c_{\mathrm{sw}}$ we rely on the calculation of ref. 13. It has also been calculated in perturbation theory to one loop 14, 15. The coefficients $c_{\mathrm{t}}$ and $\tilde{c}_{\mathrm{t}}$ are known only in perturbation theory, to NLO [16] and LO [15] respectively:

$$
\begin{aligned}
& c_{\mathrm{t}}\left(g_{0}^{2}\right)=1-0.089 g_{0}^{2}-0.030 g_{0}^{4}, \\
& \tilde{c}_{\mathrm{t}}\left(g_{0}^{2}\right)=1-0.018 g_{0}^{2} .
\end{aligned}
$$

A more detailed discussion of perturbative $\mathrm{O}(a)$ improvement will be presented in Section 4. Here we outline the main expectations related to cutoff effects, in the spirit of the Symanzik improvement programme [17]: in the absence of improvement counterterms (i.e. $c_{\mathrm{Sw}}=0$ and $c_{\mathrm{t}}=\tilde{c}_{\mathrm{t}}=1$ ), correlation functions (such as $f_{\mathrm{P}}$ and $f_{\mathrm{A}}$ ), computed at fixed UV cutoff $a^{-1}$ and renormalised non-perturbatively, should exhibit $\mathrm{O}(a)$ deviations from their continuum limit. If all improvement coefficients were known non-perturbatively, the discretisation errors would be $\mathrm{O}\left(a^{2}\right)$. With the improvement coefficients set to their tree-level values (i.e. $c_{\mathrm{sw}}=c_{\mathrm{t}}=\tilde{c}_{\mathrm{t}}=1$ ), the dominant discretisation effects are expected to be $\mathrm{O}\left(g_{0}^{2} a\right)$ and $\mathrm{O}\left(a^{2}\right)$; with one-loop coefficients we have $\mathrm{O}\left(g_{0}^{4} a\right)$ and $\mathrm{O}\left(a^{2}\right)$ errors etc. These statements refer to the chiral limit (away from which, we must also take into consideration counterterms proportional to the quark mass). Since we are in the framework of mass independent renormalisation, working in the chiral limit is adequate for our purposes. We have performed numerical simulations in two regimes:

(i) What we call "unimproved action results" (or "unimproved case" for short) consists in setting $c_{\mathrm{sw}}=0$. Moreover, we set $\tilde{c}_{\mathrm{t}}=1$, while the one-loop value ${ }^{2}$ (eq. (2.4) truncated to $\left.\mathrm{O}\left(g_{0}^{2}\right)\right)$ is used for $c_{\mathrm{t}}$. Since the action in the lattice bulk is unimproved, the dominant discretisation effects ought to be $\mathrm{O}(a)$.

(ii) What we call "improved action results" (or "improved case" for short) consists in using the Clover action with a non-perturbative $c_{\mathrm{sw}}$. The one-loop value

\footnotetext{
${ }^{2}$ This is a choice of convenience: it is important to know for renormalisation purposes (see eq. (3.3) below) the dependence of the Schrödinger functional renormalised coupling $\bar{g}(1 / L)$ on the bare coupling $g_{0}$. This dependence is known non-perturbatively 112 for the pure Yang-Mills action with this $c_{\mathrm{t}}$ value. In any case, the choice for $c_{\mathrm{t}}$ has no bearing on the order of leading lattice artifacts.
} 
from eqs. (2.4) and (2.5) is used for $c_{\mathrm{t}}$ and $\tilde{c}_{\mathrm{t}}$ respectively. Thus the dominant discretisation errors should be $\mathrm{O}\left(g_{0}^{4} a\right)$ and $\mathrm{O}\left(a^{2}\right)$. Since the former only arise from perturbatively improved boundary counterterms, while everything in the lattice bulk is fully improved, it is reasonable to expect that correlation functions are mostly affected by $\mathrm{O}\left(a^{2}\right)$ errors. Numerical support for this expectation has been presented in ref. [2].

\section{The step scaling function}

SF renormalisation schemes are mass independent; i.e. simulations can be performed in the chiral limit. The renormalisation scale is set at the lattice IR cutoff (i.e. $\mu=1 / L)$; the renormalised coupling $\bar{g}(1 / L)$ and quark mass $\bar{m}(1 / L)$ are then only functions of $L$. The SF renormalised coupling has been defined in ref. 1]. The renormalised quark mass is [2]

$$
\bar{m}(1 / L)=\lim _{a \rightarrow 0} Z_{\mathrm{A}}\left(g_{0}\right) Z_{\mathrm{P}}^{-1}\left(g_{0}, L / a\right) m\left(g_{0}\right)
$$

where $m\left(g_{0}\right)$ is defined in eq. (2.1) and the renormalisation condition for the pseudoscalar operator is

$$
\left.Z_{\mathrm{P}}\left(g_{0}, L / a\right) \frac{f_{\mathrm{P}}(L / 2)}{\sqrt{f_{1}}}\right|_{m=0}=c(\theta, a / L),
$$

with $c(\theta, a / L)$ such that at tree level $Z_{\mathrm{P}}(0, L / a)=1$. We will always impose eq. (3.2) at $\theta=0.5$ 2, 18, and hence eliminate any explicit reference to $\theta$ from now on. The axial current normalisation $Z_{\mathrm{A}}\left(g_{0}\right)$, being scale independent, has no effect on the renormalisation group running of the quark mass; thus it is of no immediate consequence to the present work.

Here we are interested in the step scaling function of the quark mass, which is defined in the chiral limit $m\left(g_{0}\right)=0$, for a lattice of a given resolution $L / a$ and at fixed renormalised coupling $\bar{g}^{2}(1 / L)=u$, by

$$
\Sigma_{\mathrm{P}}(u, a / L)=\left.\frac{Z_{\mathrm{P}}\left(g_{0}, 2 L / a\right)}{Z_{\mathrm{P}}\left(g_{0}, L / a\right)}\right|_{m=0, \bar{g}^{2}(1 / L)=u} .
$$

This quantity is finite in the continuum limit

$$
\sigma_{\mathrm{P}}(u)=\lim _{a \rightarrow 0} \Sigma_{\mathrm{P}}(u, a / L)=\left.\frac{\bar{m}(1 / L)}{\bar{m}(1 /(2 L))}\right|_{\bar{g}^{2}(1 / L)=u} .
$$


The physical meaning of $\sigma_{\mathrm{P}}$ follows from the RG equation obeyed by the renormalised quark mass

$$
\mu \frac{\partial \bar{m}(\mu)}{\partial \mu}=\tau(\bar{g}(\mu)) \bar{m}(\mu)
$$

(recall that $\mu=1 / L$ ). Upon integration of this equation between scales $L^{-1}$ and $(2 L)^{-1}$ we obtain

$$
\sigma_{\mathrm{P}}(u)=\exp \left\{-\int_{\bar{g}(1 / L)}^{\bar{g}(1 /(2 L))} \frac{\tau(g)}{\beta(g)} \mathrm{d} g\right\},
$$

with $\beta(g)$ the Callan-Symanzik function. Thus, $\sigma_{\mathrm{P}}$ is closely related to the quark mass anomalous dimension.

The lattice SSF $\Sigma_{\mathrm{P}}$ is not unique: it depends on the details of the lattice regularisation (e.g. the type of lattice action chosen, the level of $\mathrm{O}(a)$ improvement etc.). Its continuum limit, however, should be unique (i.e. universality should hold), unless lattice QCD, or at least the specific regularisation implemented here, exhibits some unexpected pathology. This is what the present paper has set out to explore, in the spirit of refs. [5, 6].

\section{Discretisation effects in perturbation theory}

The expansion of the SSF in renormalised perturbation theory reads

$$
\sigma_{\mathrm{P}}(u)=1+\sum_{n=1}^{\infty} \sigma_{\mathrm{P}}^{(n)} u^{n},
$$

with the LO universal RG coefficient $\sigma_{\mathrm{P}}^{(1)}=-8 \ln (2) /(4 \pi)^{2}$. In perturbation theory the cutoff dependence of the SSF can be studied by expanding

$$
\frac{\Sigma_{\mathrm{P}}(u, a / L)}{\sigma_{\mathrm{P}}(u)}=1+\sum_{n=0}^{\infty} k_{n}(a / L) u^{n} .
$$

Note that due to the choice of renormalisation condition (3.2), discretisation errors are absent at tree level (i.e. $k_{0}(a / L)=0$ ). Moreover, $k_{n}(0)=0$ by construction. The quantity $k_{1}(a / L)$, which contains the cutoff effects at one loop, is known for the improved case from the work of Sint and Weisz [18] for $L / a=4,6, \ldots, 16$. In their notation, it is given by

$$
k_{1}(a / L)=k(\infty) \delta_{k}(L / a)=-\frac{8 \ln (2)}{(4 \pi)^{2}} \delta_{k}(L / a)
$$




\begin{tabular}{crr}
\hline$L / a$ & $k_{1}$ (Unimproved) & \multicolumn{1}{c}{$k_{1}$ (Improved) } \\
\hline 4 & $1.5366198 \times 10^{-3}$ & $-7.4992105 \times 10^{-3}$ \\
6 & $8.1921362 \times 10^{-4}$ & $-7.2993963 \times 10^{-4}$ \\
8 & $1.2463902 \times 10^{-4}$ & $9.2204726 \times 10^{-5}$ \\
10 & $-2.3064991 \times 10^{-4}$ & $2.1695501 \times 10^{-4}$ \\
12 & $-4.0199062 \times 10^{-4}$ & $2.2359743 \times 10^{-4}$ \\
14 & $-4.8254066 \times 10^{-4}$ & $2.0536785 \times 10^{-4}$ \\
16 & $-5.1712918 \times 10^{-4}$ & $1.8293858 \times 10^{-4}$ \\
18 & $-5.2761682 \times 10^{-4}$ & $1.6171688 \times 10^{-4}$ \\
20 & $-5.2516423 \times 10^{-4}$ & $1.4302219 \times 10^{-4}$ \\
22 & $-5.1573079 \times 10^{-4}$ & $1.2694608 \times 10^{-4}$ \\
24 & $-5.0261759 \times 10^{-4}$ & $1.1321941 \times 10^{-4}$ \\
26 & $-4.8770374 \times 10^{-4}$ & $1.0149790 \times 10^{-4}$ \\
\hline
\end{tabular}

Table 1: Results for the cutoff dependence of the step scaling function of the pseudoscalar density at one loop in perturbation theory.

with $\delta_{k}(L / a)$ tabulated in Table 2 of ref. [18] (the case of interest to us is $\theta=$ $0.5, \rho=T / L=1)$. We have repeated these calculations for the unimproved case and extended both cases to $L / a=18,20, \ldots, 26$. The results are summarised in Table 1 and Fig. 1 .

The present perturbative analysis has been motivated by the wish to explore in detail the cutoff dependence of our non-perturbative estimates of $\Sigma_{\mathrm{P}}(u, a / L)$, obtained in our simulations at four lattice resolutions $L / a=6,8,12,16$ (see next Section). Strictly speaking we therefore need to know $k_{1}(a / L)$ only at these four values of $(a / L)$, for both the unimproved and improved cases. However it is clear that one-loop cutoff effects can have a rather non-trivial overall behaviour as $(a / L)$ is reduced. Had we limited our perturbative calculation to the range of interest (i.e. $L / a=6,8,12,16)$, in the unimproved case we would have only observed that $k_{1}$ crosses over monotonically the abscissa axis at about $L / a=8$, without signalling that it indeed converges towards its limiting value $k_{1}(0)=0$ (the improved case already "bends over" towards the origin within this range). As a partial safeguard against the eventuality of some uncontrolled error afflicting our perturbative results (e.g. rounding in the numerical integrations), we have extended the calculations all the way to $L / a=26$. Table 1 and Fig. 1 demonstrate that indeed $k_{1}$ reaches a local extremum and subsequently points towards the origin of the axes, as it should.

In order to gain some further insight into this behaviour, we recall that the 
coefficients $k_{n}$ of the perturbative series can be expanded as

$$
k_{n}(a / L)=\sum_{p=1}^{\infty}\left(\frac{a}{L}\right)^{p} \sum_{l=0}^{n} c_{p l}^{(n)}[\ln (a / L)]^{l},
$$

with the leading $\mathrm{O}(u)$ (one-loop) discretisation effects having the form

$$
\begin{aligned}
k_{1}(a / L)= & \frac{a}{L}\left[c_{10}^{(1)}+c_{11}^{(1)} \ln (a / L)\right] \\
& +\frac{a^{2}}{L^{2}}\left[c_{20}^{(1)}+c_{21}^{(1)} \ln (a / L)\right]+\mathrm{O}\left(a^{3} / L^{3}\right) .
\end{aligned}
$$

Tree-level improvement implies in general that $c_{1 n}^{(n)}=0$; thus the $\mathrm{O}(a / L)$ one-loop perturbative contribution is as above, but without the logarithm (i.e. $c_{11}^{(1)}=0$ ). One-loop improvement implies that $c_{1 n}^{(n)}=c_{1, n-1}^{(n)}=0$; thus the $\mathrm{O}(a / L)$ one-loop perturbative contributions of eq. (4.5) all vanish $\left(c_{10}^{(1)}=c_{11}^{(1)}=0\right)$. The leading discretisation effects of $\Sigma_{\mathrm{P}}$ are then $\mathrm{O}\left(u a^{2} / L^{2}\right)$. Such "dominant" discretisation effects may in practice compete with the next order $\mathrm{O}\left(u^{2} a / L\right)$ errors, arising from $k_{2}(a / L)$. In all cases the functional form of $k_{1}(a / L)$ is clearly complicated; the observed behaviour can be explained by the strengths (and relative signs) of the various coefficients $c_{p l}^{(n)}$.

\section{Non-perturbative computation of the step scaling function}

In this Section we study the extrapolation of $\Sigma_{\mathrm{P}}$ to the continuum limit. We also obtain a very accurate estimate of the ratio of the RGI quark mass to its renormalised counterpart at a hadronic scale. The method of computation is identical to that of ref. [2].

\subsection{Continuum limit of the step scaling function}

For both the unimproved and improved cases the lattice SSF $\Sigma_{\mathrm{P}}$ has been evaluated at 14 values of the renormalised coupling $\bar{g}(1 / L)$, each for four lattice resolutions $L / a=6,8,12$ and 16 . Note that in ref. [2] only the improved case has been studied. A full collection of our raw data is presented in Tables 2 and 3 . The "tuning" of $\beta$ at the four $L / a$ values, corresponding to an (almost) fixed renormalised coupling $\bar{g}^{2}(1 / L)=u$ has been taken over from ref. [2]. This corresponds to the first three columns of Tables 2 and 3. The same is true of $\kappa_{\mathrm{c}}$ for the improved case (fourth column of Table 2). All other results are new ${ }^{3}$. In the strong coupling regime new

\footnotetext{
${ }^{3}$ The computation of $\kappa_{\mathrm{c}}$ for the unimproved case was first performed in ref. 8 for 9 of the 14 couplings used here; following [2], this is done at $\theta=0$.
} 


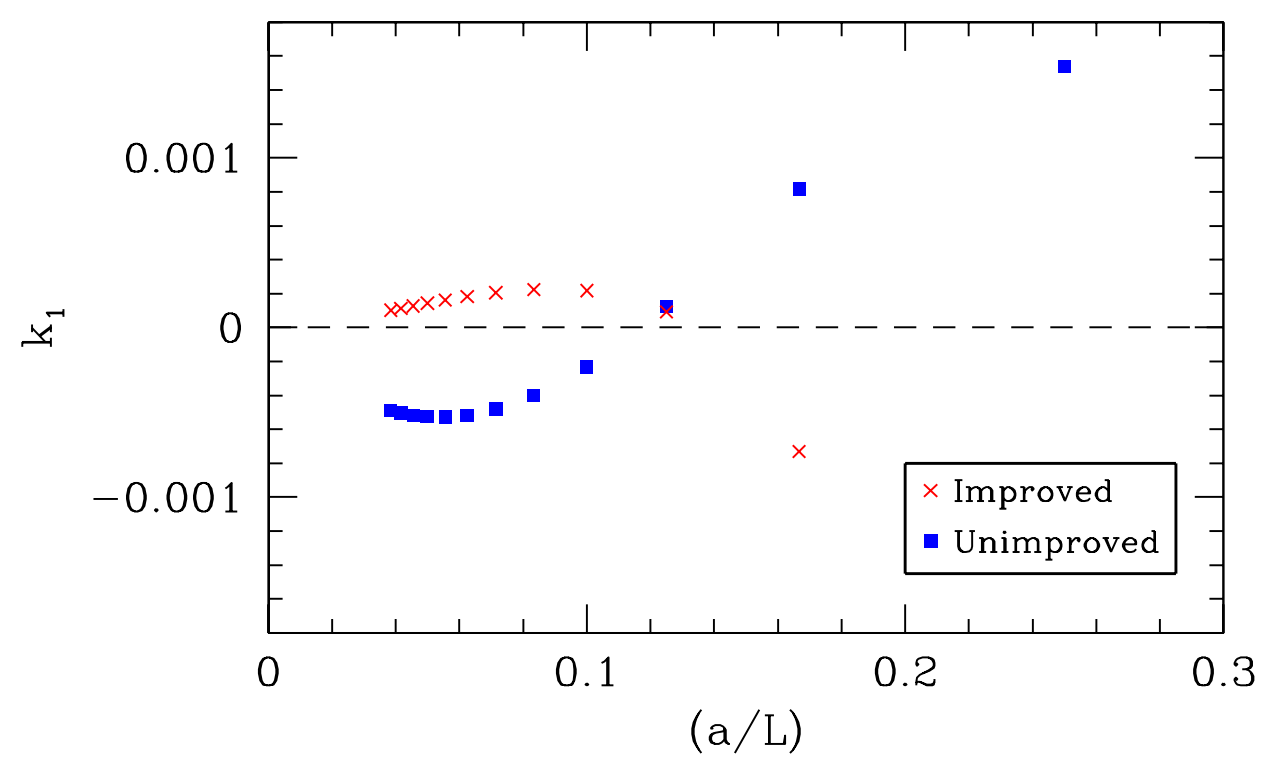

Figure 1: Cutoff dependence of the step scaling function of the pseudoscalar density at one loop in perturbation theory.

results have been obtained at $\bar{g}^{2}(1 / L)=3.111$. The statistical accuracy of our improved and unimproved $\Sigma_{\mathrm{P}}(u, a / L)$ results is comparable (save for a few cases where the improved data has somewhat smaller errors). ${ }^{4}$

A comparison of our data in the improved case with those of ref. 22 reveals fairly compatible results: for $Z_{\mathrm{P}}\left(g_{0}, L / a\right)$ and $Z_{\mathrm{P}}\left(g_{0}, 2 L / a\right)$ we mostly agree within errors, save for a few cases in which agreement is within $2 \sigma$; the same is true for $\Sigma_{\mathrm{P}}(u, a / L)$, with some data being compatible only within $1.5 \sigma$.

If quenched lattice QCD has a universal continuum limit, then both sets of $\Sigma_{\mathrm{P}}$ results (improved and unimproved action) ought to extrapolate to the same continuum value $\sigma_{\mathrm{P}}$ at fixed coupling $u$. What we have set out to investigate is the power dependence (linear and/or quadratic) of the results on $(a / L)$. From the analysis of ref. [2] we expect the dominant discretisation effects to be $\mathrm{O}(a)$ in the unimproved case and $\mathrm{O}\left(a^{2}\right)$ in the improved one. Nevertheless we have performed fits on both datasets with the two Ansätze

$$
\begin{aligned}
& \Sigma_{\mathrm{P}}(u, a / L)=\sigma_{\mathrm{P}}(u)+\rho(u)(a / L), \\
& \Sigma_{\mathrm{P}}(u, a / L)=\sigma_{\mathrm{P}}(u)+\rho(u)(a / L)^{2} .
\end{aligned}
$$

\footnotetext{
${ }^{4}$ The typical statistics accumulated for small lattices is of several hundred configurations. For the largest lattices the number of configurations ranges from around 60 at the weakest couplings to around 200 at the strongest ones.
} 


\begin{tabular}{rrlllll}
\hline$\beta$ & $\frac{L}{a}$ & $\bar{g}^{2}(1 / L)$ & \multicolumn{1}{c}{$\kappa_{\mathrm{C}}$} & $Z_{\mathrm{P}}\left(g_{0}, \frac{L}{a}\right)$ & $Z_{\mathrm{P}}\left(g_{0}, \frac{2 L}{a}\right)$ & $\Sigma_{\mathrm{P}}\left(u, \frac{a}{L}\right)$ \\
\hline 10.7503 & 6 & $0.8873(5)$ & $0.130591(4)$ & $0.8480(5)$ & $0.8192(8)$ & $0.9660(11)$ \\
11.0000 & 8 & $0.8873(10)$ & $0.130439(3)$ & $0.8402(5)$ & $0.8125(10)$ & $0.9670(13)$ \\
11.3384 & 12 & $0.8873(30)$ & $0.130251(2)$ & $0.8331(8)$ & $0.8049(11)$ & $0.9662(16)$ \\
11.5736 & 16 & $0.8873(25)$ & $0.130125(2)$ & $0.8253(8)$ & $0.7986(15)$ & $0.9676(20)$ \\
10.0500 & 6 & $0.9944(7)$ & $0.131073(5)$ & $0.8326(5)$ & $0.8012(8)$ & $0.9623(11)$ \\
10.3000 & 8 & $0.9944(13)$ & $0.130889(3)$ & $0.8260(7)$ & $0.7957(9)$ & $0.9633(14)$ \\
10.6086 & 12 & $0.9944(30)$ & $0.130692(2)$ & $0.8153(8)$ & $0.7826(13)$ & $0.9599(19)$ \\
10.8910 & 16 & $0.9944(28)$ & $0.130515(2)$ & $0.8102(7)$ & $0.7796(15)$ & $0.9622(20)$ \\
9.5030 & 6 & $1.0989(8)$ & $0.131514(5)$ & $0.8200(6)$ & $0.7831(10)$ & $0.9550(14)$ \\
9.7500 & 8 & $1.0989(13)$ & $0.131312(3)$ & $0.8117(6)$ & $0.7769(9)$ & $0.9571(13)$ \\
10.0577 & 12 & $1.0989(40)$ & $0.131079(3)$ & $0.8005(9)$ & $0.7668(11)$ & $0.9579(17)$ \\
10.3419 & 16 & $1.0989(44)$ & $0.130876(2)$ & $0.7959(10)$ & $0.7630(11)$ & $0.9587(18)$ \\
8.8997 & 6 & $1.2430(13)$ & $0.132072(9)$ & $0.8013(4)$ & $0.7633(8)$ & $0.9526(11)$ \\
9.1544 & 8 & $1.2430(14)$ & $0.131838(4)$ & $0.7945(5)$ & $0.7548(11)$ & $0.9500(15)$ \\
9.5202 & 12 & $1.2430(35)$ & $0.131503(3)$ & $0.7842(7)$ & $0.7498(11)$ & $0.9561(16)$ \\
9.7350 & 16 & $1.2430(34)$ & $0.131335(3)$ & $0.7774(11)$ & $0.7407(14)$ & $0.9528(22)$ \\
8.6129 & 6 & $1.3293(12)$ & $0.132380(6)$ & $0.7909(6)$ & $0.7501(11)$ & $0.9484(16)$ \\
8.8500 & 8 & $1.3293(21)$ & $0.132140(5)$ & $0.7826(7)$ & $0.7435(11)$ & $0.9500(16)$ \\
9.1859 & 12 & $1.3293(60)$ & $0.131814(3)$ & $0.7738(10)$ & $0.7348(15)$ & $0.9496(23)$ \\
9.4381 & 16 & $1.3293(40)$ & $0.131589(2)$ & $0.7661(9)$ & $0.7273(19)$ & $0.9494(27)$ \\
8.3124 & 6 & $1.4300(20)$ & $0.132734(10)$ & $0.7808(5)$ & $0.7356(8)$ & $0.9421(12)$ \\
8.5598 & 8 & $1.4300(21)$ & $0.132453(5)$ & $0.7727(6)$ & $0.7282(11)$ & $0.9424(16)$ \\
8.9003 & 12 & $1.4300(50)$ & $0.132095(3)$ & $0.7621(10)$ & $0.7195(14)$ & $0.9441(22)$ \\
9.1415 & 16 & $1.4300(58)$ & $0.131855(3)$ & $0.7551(8)$ & $0.7129(16)$ & $0.9441(23)$ \\
7.9993 & 6 & $1.5553(15)$ & $0.133118(7)$ & $0.7659(4)$ & $0.7178(12)$ & $0.9372(16)$ \\
8.2500 & 8 & $1.5553(24)$ & $0.132821(5)$ & $0.7575(7)$ & $0.7127(12)$ & $0.9409(18)$ \\
8.5985 & 12 & $1.5533(70)$ & $0.132427(3)$ & $0.7484(11)$ & $0.7021(14)$ & $0.9381(23)$ \\
8.8323 & 16 & $1.5533(70)$ & $0.132169(3)$ & $0.7405(11)$ & $0.6966(19)$ & $0.9407(29)$ \\
\hline & & & & & & \\
\hline
\end{tabular}

Table 2: Results for the step scaling function $\Sigma_{\mathrm{P}}$, improved case. 


\begin{tabular}{rrlllll}
\hline$\beta$ & $\frac{L}{a}$ & $\bar{g}^{2}(1 / L)$ & \multicolumn{1}{c}{$\kappa_{\mathrm{C}}$} & $Z_{\mathrm{P}}\left(g_{0}, \frac{L}{a}\right)$ & $Z_{\mathrm{P}}\left(g_{0}, \frac{2 L}{a}\right)$ & $\Sigma_{\mathrm{P}}\left(u, \frac{a}{L}\right)$ \\
\hline 7.7170 & 6 & $1.6950(26)$ & $0.133517(8)$ & $0.7527(6)$ & $0.6997(4)$ & $0.9296(9)$ \\
7.9741 & 8 & $1.6950(28)$ & $0.133179(5)$ & $0.7452(6)$ & $0.6934(11)$ & $0.9305(17)$ \\
8.3218 & 12 & $1.6950(79)$ & $0.132756(4)$ & $0.7353(4)$ & $0.6858(14)$ & $0.9327(20)$ \\
8.5479 & 16 & $1.6950(90)$ & $0.132485(3)$ & $0.7266(12)$ & $0.6792(16)$ & $0.9348(27)$ \\
7.4082 & 6 & $1.8811(22)$ & $0.133961(8)$ & $0.7345(7)$ & $0.6773(5)$ & $0.9221(11)$ \\
7.6547 & 8 & $1.8811(28)$ & $0.133632(6)$ & $0.7259(7)$ & $0.6712(12)$ & $0.9246(19)$ \\
7.9993 & 12 & $1.8811(38)$ & $0.133159(4)$ & $0.7174(4)$ & $0.6630(13)$ & $0.9242(19)$ \\
8.2415 & 16 & $1.8811(99)$ & $0.132847(3)$ & $0.7132(16)$ & $0.6578(14)$ & $0.9223(29)$ \\
7.1214 & 6 & $2.1000(39)$ & $0.134423(9)$ & $0.7149(7)$ & $0.6512(5)$ & $0.9109(11)$ \\
7.3632 & 8 & $2.1000(45)$ & $0.134088(6)$ & $0.7069(6)$ & $0.6452(13)$ & $0.9127(20)$ \\
7.6985 & 12 & $2.1000(80)$ & $0.133599(4)$ & $0.6976(4)$ & $0.6370(14)$ & $0.9131(21)$ \\
7.9560 & 16 & $2.100(11)$ & $0.133229(3)$ & $0.6904(12)$ & $0.6348(12)$ & $0.9195(24)$ \\
6.7807 & 6 & $2.4484(37)$ & $0.134994(11)$ & $0.6874(8)$ & $0.6112(5)$ & $0.8891(13)$ \\
7.0197 & 8 & $2.4484(45)$ & $0.134639(7)$ & $0.6796(7)$ & $0.6079(14)$ & $0.8945(23)$ \\
7.3551 & 12 & $2.4484(80)$ & $0.134141(5)$ & $0.6711(5)$ & $0.5978(15)$ & $0.8908(23)$ \\
7.6101 & 16 & $2.448(17)$ & $0.133729(4)$ & $0.6664(12)$ & $0.5996(13)$ & $0.8998(25)$ \\
6.5512 & 6 & $2.770(7)$ & $0.135327(12)$ & $0.6628(8)$ & $0.5775(4)$ & $0.8713(12)$ \\
6.7860 & 8 & $2.770(7)$ & $0.135056(8)$ & $0.6551(8)$ & $0.5753(14)$ & $0.8782(24)$ \\
7.1190 & 12 & $2.770(11)$ & $0.134513(5)$ & $0.6487(5)$ & $0.5704(10)$ & $0.8793(17)$ \\
7.3686 & 16 & $2.770(14)$ & $0.134114(3)$ & $0.6452(14)$ & $0.5672(15)$ & $0.8791(30)$ \\
6.3665 & 6 & $3.111(4)$ & $0.135488(6)$ & $0.6395(9)$ & $0.5427(13)$ & $0.8486(24)$ \\
6.6100 & 8 & $3.111(6)$ & $0.135339(3)$ & $0.6356(8)$ & $0.5466(15)$ & $0.8600(26)$ \\
6.9322 & 12 & $3.111(12)$ & $0.134855(3)$ & $0.6290(12)$ & $0.5363(15)$ & $0.8526(29)$ \\
7.1911 & 16 & $3.111(16)$ & $0.134411(3)$ & $0.6286(9)$ & $0.5438(16)$ & $0.8651(28)$ \\
6.2204 & 6 & $3.480(8)$ & $0.135470(15)$ & $0.6179(4)$ & $0.5058(12)$ & $0.8186(20)$ \\
6.4527 & 8 & $3.480(14)$ & $0.135543(9)$ & $0.6129(5)$ & $0.5085(17)$ & $0.8297(29)$ \\
6.7750 & 12 & $3.480(39)$ & $0.135121(5)$ & $0.6092(10)$ & $0.5102(15)$ & $0.8375(28)$ \\
7.0203 & 16 & $3.480(21)$ & $0.134707(4)$ & $0.6050(10)$ & $0.5056(17)$ & $0.8357(31)$ \\
\hline & & & & & & \\
\hline
\end{tabular}

Table 2: (continued) 


\begin{tabular}{rrlllll}
\hline$\beta$ & $\frac{L}{a}$ & $\bar{g}^{2}(1 / L)$ & \multicolumn{1}{c}{$\kappa_{\mathrm{C}}$} & $Z_{\mathrm{P}}\left(g_{0}, \frac{L}{a}\right)$ & $Z_{\mathrm{P}}\left(g_{0}, \frac{2 L}{a}\right)$ & $\Sigma_{\mathrm{P}}\left(u, \frac{a}{L}\right)$ \\
\hline 10.7503 & 6 & $0.8873(5)$ & $0.134696(7)$ & $0.8559(5)$ & $0.8290(7)$ & $0.9686(10)$ \\
11.0000 & 8 & $0.8873(10)$ & $0.134548(6)$ & $0.8450(5)$ & $0.8188(8)$ & $0.9690(11)$ \\
11.3384 & 12 & $0.8873(30)$ & $0.134277(5)$ & $0.8336(6)$ & $0.8066(10)$ & $0.9676(14)$ \\
11.5736 & 16 & $0.8873(25)$ & $0.134068(6)$ & $0.8264(7)$ & $0.8003(13)$ & $0.9684(18)$ \\
10.0500 & 6 & $0.9944(7)$ & $0.135659(8)$ & $0.8413(5)$ & $0.8123(8)$ & $0.9655(11)$ \\
10.3000 & 8 & $0.9944(13)$ & $0.135457(5)$ & $0.8310(5)$ & $0.8012(9)$ & $0.9641(12)$ \\
10.6086 & 12 & $0.9944(30)$ & $0.135160(4)$ & $0.8188(7)$ & $0.7887(12)$ & $0.9632(17)$ \\
10.8910 & 16 & $0.9944(28)$ & $0.134849(6)$ & $0.8108(8)$ & $0.7826(16)$ & $0.9652(22)$ \\
9.5030 & 6 & $1.0989(8)$ & $0.136520(5)$ & $0.8292(6)$ & $0.7973(8)$ & $0.9615(12)$ \\
9.7500 & 8 & $1.0989(13)$ & $0.136310(3)$ & $0.8189(5)$ & $0.7847(9)$ & $0.9582(12)$ \\
10.0577 & 12 & $1.0989(40)$ & $0.135949(4)$ & $0.8060(8)$ & $0.7739(11)$ & $0.9602(17)$ \\
10.3419 & 16 & $1.0989(44)$ & $0.135572(4)$ & $0.7980(12)$ & $0.7641(11)$ & $0.9575(20)$ \\
8.8997 & 6 & $1.2430(13)$ & $0.137706(5)$ & $0.8119(6)$ & $0.7775(8)$ & $0.9576(12)$ \\
9.1544 & 8 & $1.2430(14)$ & $0.137400(4)$ & $0.8009(6)$ & $0.7651(9)$ & $0.9553(13)$ \\
9.5202 & 12 & $1.2430(35)$ & $0.136855(2)$ & $0.7880(8)$ & $0.7521(12)$ & $0.9544(18)$ \\
9.7350 & 16 & $1.2430(34)$ & $0.136523(4)$ & $0.7805(9)$ & $0.7452(14)$ & $0.9548(21)$ \\
8.6129 & 6 & $1.3293(12)$ & $0.138346(6)$ & $0.8045(7)$ & $0.7654(8)$ & $0.9514(13)$ \\
8.8500 & 8 & $1.3293(21)$ & $0.138057(4)$ & $0.7912(6)$ & $0.7525(10)$ & $0.9511(15)$ \\
9.1859 & 12 & $1.3293(60)$ & $0.137503(2)$ & $0.7779(9)$ & $0.7378(12)$ & $0.9485(19)$ \\
9.4381 & 16 & $1.3293(40)$ & $0.137061(4)$ & $0.7703(13)$ & $0.7286(15)$ & $0.9459(25)$ \\
8.3124 & 6 & $1.4300(20)$ & $0.139128(11)$ & $0.7905(7)$ & $0.7517(9)$ & $0.9509(14)$ \\
8.5598 & 8 & $1.4300(21)$ & $0.138742(7)$ & $0.7800(6)$ & $0.7377(11)$ & $0.9458(16)$ \\
8.9003 & 12 & $1.4300(50)$ & $0.138120(8)$ & $0.7669(10)$ & $0.7262(17)$ & $0.9469(25)$ \\
9.1415 & 16 & $1.4300(58)$ & $0.137655(5)$ & $0.7586(9)$ & $0.7190(17)$ & $0.9478(25)$ \\
7.9993 & 6 & $1.5553(15)$ & $0.140003(11)$ & $0.7808(7)$ & $0.7350(9)$ & $0.9413(14)$ \\
8.2500 & 8 & $1.5553(24)$ & $0.139588(8)$ & $0.7671(6)$ & $0.7237(11)$ & $0.9434(16)$ \\
8.5985 & 12 & $1.5533(70)$ & $0.138847(6)$ & $0.7560(9)$ & $0.7083(16)$ & $0.9369(24)$ \\
8.8323 & 16 & $1.5533(70)$ & $0.138339(7)$ & $0.7458(13)$ & $0.6992(18)$ & $0.9375(29)$ \\
\hline & & & & & \\
\hline
\end{tabular}

Table 3: Results for the step scaling function $\Sigma_{\mathrm{P}}$, unimproved case. 


\begin{tabular}{rrlllll}
\hline$\beta$ & $\frac{L}{a}$ & $\bar{g}^{2}(1 / L)$ & \multicolumn{1}{c}{$\kappa_{\mathrm{C}}$} & $Z_{\mathrm{P}}\left(g_{0}, \frac{L}{a}\right)$ & $Z_{\mathrm{P}}\left(g_{0}, \frac{2 L}{a}\right)$ & $\Sigma_{\mathrm{P}}\left(u, \frac{a}{L}\right)$ \\
\hline 7.7170 & 6 & $1.6950(26)$ & $0.140954(12)$ & $0.7650(7)$ & $0.7195(9)$ & $0.9405(15)$ \\
7.9741 & 8 & $1.6950(28)$ & $0.140438(8)$ & $0.7550(7)$ & $0.7095(15)$ & $0.9397(22)$ \\
8.3218 & 12 & $1.6950(79)$ & $0.139589(6)$ & $0.7418(10)$ & $0.6940(16)$ & $0.9356(25)$ \\
8.5479 & 16 & $1.6950(90)$ & $0.139058(6)$ & $0.7328(11)$ & $0.6823(19)$ & $0.9311(29)$ \\
7.4082 & 6 & $1.8811(22)$ & $0.142145(11)$ & $0.7489(7)$ & $0.6994(10)$ & $0.9339(16)$ \\
7.6547 & 8 & $1.8811(28)$ & $0.141572(9)$ & $0.7368(7)$ & $0.6829(13)$ & $0.9268(20)$ \\
7.9993 & 12 & $1.8811(38)$ & $0.140597(6)$ & $0.7241(11)$ & $0.6725(15)$ & $0.9287(25)$ \\
8.2415 & 16 & $1.8811(99)$ & $0.139900(6)$ & $0.7161(12)$ & $0.6652(16)$ & $0.9289(27)$ \\
7.1214 & 6 & $2.1000(39)$ & $0.143416(11)$ & $0.7309(8)$ & $0.6746(10)$ & $0.9230(17)$ \\
7.3632 & 8 & $2.1000(45)$ & $0.142749(9)$ & $0.7181(7)$ & $0.6564(17)$ & $0.9141(25)$ \\
7.6985 & 12 & $2.1000(80)$ & $0.141657(6)$ & $0.7037(8)$ & $0.6440(13)$ & $0.9152(21)$ \\
7.9560 & 16 & $2.100(11)$ & $0.140817(7)$ & $0.6980(12)$ & $0.6399(15)$ & $0.9168(27)$ \\
6.7807 & 6 & $2.4484(37)$ & $0.145286(11)$ & $0.7057(8)$ & $0.6403(11)$ & $0.9073(19)$ \\
7.0197 & 8 & $2.4484(45)$ & $0.144454(7)$ & $0.6921(8)$ & $0.6224(12)$ & $0.8993(20)$ \\
7.3551 & 12 & $2.4484(80)$ & $0.143113(6)$ & $0.6796(8)$ & $0.6065(19)$ & $0.8924(30)$ \\
7.6101 & 16 & $2.448(17)$ & $0.142107(6)$ & $0.6745(12)$ & $0.6095(19)$ & $0.9036(32)$ \\
6.5512 & 6 & $2.770(7)$ & $0.146825(11)$ & $0.6839(9)$ & $0.6083(11)$ & $0.8895(20)$ \\
6.7860 & 8 & $2.770(7)$ & $0.145859(7)$ & $0.6702(8)$ & $0.5938(17)$ & $0.8860(27)$ \\
7.1190 & 12 & $2.770(11)$ & $0.144299(8)$ & $0.6583(11)$ & $0.5796(14)$ & $0.8804(26)$ \\
7.3686 & 16 & $2.770(14)$ & $0.143175(7)$ & $0.6532(15)$ & $0.5772(19)$ & $0.8836(35)$ \\
6.3665 & 6 & $3.111(4)$ & $0.148317(10)$ & $0.6635(9)$ & $0.5770(11)$ & $0.8696(20)$ \\
6.6100 & 8 & $3.111(6)$ & $0.147112(7)$ & $0.6529(9)$ & $0.5642(14)$ & $0.8641(25)$ \\
6.9322 & 12 & $3.111(12)$ & $0.145371(7)$ & $0.6394(11)$ & $0.5504(20)$ & $0.8608(35)$ \\
7.1911 & 16 & $3.111(16)$ & $0.144060(8)$ & $0.6329(13)$ & $0.5479(17)$ & $0.8657(32)$ \\
6.2204 & 6 & $3.480(8)$ & $0.149685(15)$ & $0.6473(10)$ & $0.5466(13)$ & $0.8444(24)$ \\
6.4527 & 8 & $3.480(14)$ & $0.148391(9)$ & $0.6309(9)$ & $0.5315(23)$ & $0.8424(38)$ \\
6.7750 & 12 & $3.480(39)$ & $0.146408(7)$ & $0.6201(9)$ & $0.5218(21)$ & $0.8415(36)$ \\
7.0203 & 16 & $3.480(21)$ & $0.145025(8)$ & $0.6131(11)$ & $0.5177(20)$ & $0.8444(36)$ \\
\hline & & & & & & \\
\hline
\end{tabular}

Table 3: (continued) 
Another issue raised in ref. [2] is the number of data points which should be included in each fit. In that work the $L / a=6$ results were dropped from the fits, being too far from the continuum limit. We have performed fits with all data (4-point fits) and also without the $L / a=6$ data (3-point fits). This means that we have applied a total of four fitting procedures (the two Ansätze of eqs. (5.15.2), each for a 3- and a 4-point fit).

The results of these fitting procedures can be summarised as follows:

(i) In all cases, the statistical accuracy of our result for $\sigma_{\mathrm{P}}$ is better than $1 \%$. The results for the linear or quadratic coefficients $\rho$ have large statistical uncertainties (up to 100\%), reflecting an overall weak cutoff dependence of $\Sigma_{P}$.

(ii) For any given lattice regularisation (i.e. improved or unimproved) and with any given fitting Ansatz (i.e. linear or quadratic in $(a / L)$ ), the results for $\sigma_{\mathrm{P}}$ obtained by a 3 -point fit are compatible to those obtained by a 4 -point fit (at fixed coupling $u$ ). Naturally, the former have a larger error.

(iii) For either lattice regularisation (i.e. improved or unimproved) and with any given number of fitting points (i.e. 3-point fit or 4-point fit) the results for $\sigma_{\mathrm{P}}$ obtained by a linear fit in $(a / L)$ are compatible to those obtained by a quadratic fit in $(a / L)$ (at fixed coupling $u$ ). There is just one exception for the improved data at the strongest coupling $u=3.480$ with a 4 -point fit (agreement is within $1.5 \sigma)$. The results from the quadratic fit are more accurate, due to the fact that the extrapolation from the range of simulated data points to the continuum limit is shorter in $(a / L)^{2}$ than in $(a / L)$.

(iv) The goodness of fit is always satisfactory $\left(\chi^{2} /\right.$ d.o.f. $\left.<3\right)$ at weak and intermediate couplings $(u \in[0.8873,1.8811])$. In a limited number of cases at stronger couplings the value tends to rise considerably, but this apparently does not depend systematically on the number of fitted points and choice of fitting Ansatz. In any case, given the small number of fitted data points, $\chi^{2} /$ d.o.f. is a goodness-of-fit criterion of relatively limited value. Instead, the total $\chi^{2} /$ d.o.f. varies between 1 and 2 , indicating satisfactory overall quality of the fits.

We conservatively consider our 3-point fit results to be our best (i.e. we drop the data computed at the largest lattice spacing) and opt for linear fits in $(a / L)$ with the unimproved case and quadratic ones with the improved one. The results for these options are shown in Fig. 2 .

One could attempt to enrich this analysis along the lines of ref. [6]: we recall that the discretisation effects known from perturbation theory (see eq. (4.2) and the related discussion) can be divided out of the lattice SSF, by defining the quantity

$$
\Sigma_{\mathrm{P}}^{(2)}(u, a / L)=\frac{\Sigma_{\mathrm{P}}(u, a / L)}{1+u k_{1}(a / L)} .
$$


The continuum limit of $\Sigma_{\mathrm{P}}^{(2)}$ is trivially the same as that of $\Sigma_{\mathrm{P}}$, but the former quantity may approach it faster, as it has discretisation errors which are of order $u^{2}$. However we have seen in the previous Section that $k_{1}(a / L)$ is always numerically very small. Thus the denominator of eq. (5.3) has an imperceptible impact on $\Sigma_{\mathrm{P}}$.

The continuum extrapolations of $\Sigma_{\mathrm{P}}$ (obtained with improved and unimproved lattice actions) give results which are fully compatible both in the weak and strong coupling regions. At intermediate couplings we only have agreement within $1.5 \sigma$; see Fig. 2. The previous fitting analysis strongly suggests that this small discrepancy, rather than signalling a lack of continuum limit universality, is to be attributed to discretisation effects not being fully under control.

We will now corroborate this conclusion, by fitting our best results for the continuum SSF $\sigma_{\mathrm{P}}(u)$ with the polynomial

$$
\sigma_{\mathrm{P}}(u)=1+\sum_{n=1}^{N} s_{n} u^{n} .
$$

In all cases the first order coefficient is fixed to its PT value, $s_{1}=-8 \ln (2) /(4 \pi)^{2}$. One-parameter fits with $N=2$ yield

$$
\begin{array}{lll}
s_{2}=-0.0029(2) & \left(\chi^{2} / \text { d.o.f. } \sim 1.0\right) & \text { improved case }, \\
s_{2}=-0.0028(3) & \left(\chi^{2} / \text { d.o.f. } \sim 1.1\right) & \text { unimproved case },
\end{array}
$$

which are not too far from the PT value $s_{2}=-0.002031(4)$ of ref. [18. Oneparameter fits with $s_{2}$ fixed by PT and $N=3$ yield

$$
\begin{array}{lll}
s_{3}=-0.00031(5) & \left(\chi^{2} / \text { d.o.f. } \sim 0.7\right) & \text { improved case }, \\
s_{3}=-0.00025(11) & \left(\chi^{2} / \text { d.o.f. } \sim 1.1\right) & \text { unimproved case } .
\end{array}
$$

The above results are compatible for the two lattice actions and thus supportive of universality. This analysis becomes unstable once we push it to two- or moreparameter fits. For instance, an $N=3$ fit with two fitting parameters $\left(s_{2}\right.$ and $\left.s_{3}\right)$ yields results with errors that range between $50 \%$ and $100 \%$, while the $N=4$ fits with either two $\left(s_{3}, s_{4}\right)$ or three fitting parameters $\left(s_{2}, s_{3}, s_{4}\right)$ estimate them with $100 \%$ uncertainty.

Having mustered adequate numerical support for universality, we follow ref. 8 and calculate $\sigma_{\mathrm{P}}(u)$ (at fixed coupling $u$ ) by combined extrapolation of the $\Sigma_{\mathrm{P}}(u, a / L)$ data from both actions, constrained to a unique continuum limit. The improved (unimproved) case is assumed to depend quadratically (linearly) on $a / L$. Results for $\sigma_{\mathrm{P}}$ obtained with 3- and 4-point fits are fully compatible at all couplings, while those for $\rho(u)$ are ill-determined, as they carry up to $100 \%$ uncertainties. The goodness of fit is mostly $\chi^{2} /$ d.o.f. $\sim 1$, except for a couple of cases where it is around 4 ; anyway its average for all couplings drops below 1 . 
The 3-point fit results for $\sigma_{\mathrm{P}}(u)$ are subsequently fitted according to eq. (5.4); with $s_{1}$ given by $\mathrm{PT}$, the case corresponding to eq. (5.5) gives

$$
s_{2}=-0.0028(1) \quad\left(\chi^{2} / \text { d.o.f. } \sim 1.1\right) \quad \text { combined case },
$$

while that of eq. (5.6) gives

$$
s_{3}=-0.00030(5) \quad\left(\chi^{2} / \text { d.o.f. } \sim 0.8\right) \quad \text { combined case } .
$$

We take the results of eqs. (5.6) and (5.8) to be our best fits. In Fig. 3 we compare the LO and NLO predictions for the SSF with our discrete non-perturbative data and the best-fit result.

\subsection{RG running of the quark mass}

Using the functional form for $\sigma_{\mathrm{P}}$ we can compute the ratio of renormalised quark masses between the minimum and maximum renormalisation scales covered by our simulations. In order to be consistent with the notation of ref. [2], we denote the former by $\left(2 L_{\max }\right)^{-1}$. The ratio in question is then obtained in two steps:

First the SSF of the gauge coupling

$$
\sigma(u)=\left.\bar{g}^{2}(1 / 2 L)\right|_{\bar{g}^{2}(1 / L)=u},
$$

computed in [1, 2, is used in order to determine the correspondence between renormalised couplings and renormalisation scales. This is done through the recursion

$$
u_{l}=\sigma\left(u_{l+1}\right),
$$

with $u_{0}=\bar{g}^{2}\left(1 / L_{\max }\right)=3.48$ the initial value ${ }^{5}$. We note in passing that this procedure is based on obtaining the SSF by fitting the results of refs. [1, 2] by a polynomial

$$
\sigma(u)=u\left[1+\sum_{n=1}^{N} \sigma_{n} u^{n}\right] .
$$

In the present analysis we have used the $N=4$ series, with $\sigma_{1}, \sigma_{2}$ fixed from PT and $\sigma_{3}, \sigma_{4}$ resulting from the fit.

Second the functional form for the SSF $\sigma_{\mathrm{P}}$ is used for this sequence of couplings in order to compute the mass ratio from the product (cf. eq. (3.4))

$$
\frac{\bar{m}\left(1 / 2 L_{\max }\right)}{\bar{m}\left(1 / 2^{-k+1} L_{\max }\right)}=\prod_{l=0}^{k-1}\left[\sigma_{\mathrm{P}}\left(u_{l}\right)\right]^{-1} .
$$

\footnotetext{
${ }^{5}$ This initial value $u_{0}=3.48$ corresponds to $L_{\max } / r_{0}=0.738(16)$; the initial calculation was performed in ref. [19] while the above result is quoted in the more recent ref. [20].
} 


\begin{tabular}{cll}
\hline Ref. & Method & $\frac{M}{\bar{m}\left(1 / 2 L_{\max }\right)}$ \\
\hline$[2]$ & Improved & $1.157(12)$ \\
This work & Improved & $1.154(9)$ \\
This work & Unimproved & $1.160(13)$ \\
This work & Combined & $1.155(9)$ \\
\hline
\end{tabular}

Table 4: Ratio of the RGI quark mass to the renormalised quark mass at scale $\mu=1 / 2 L_{\max }$. "Method" refers to the procedure used in the computation of $\sigma_{\mathrm{P}}$.

In practice the range of scales covered by our simulations is spanned in $k=7$ iteration steps.

The final step in our calculation is the computation of the ratio of the RGI quark mass $M$ to its scale dependent counterpart $\bar{m}(\mu)$; in the quenched approximation this is given by [2]

$$
\frac{M}{\bar{m}(\mu)}=\left[\frac{22}{(4 \pi)^{2}} \bar{g}^{2}(\mu)\right]^{-4 / 11} \exp \left\{-\int_{0}^{\bar{g}(\mu)} \mathrm{d} g\left[\frac{\tau(g)}{\beta(g)}-\frac{8}{11 g}\right]\right\} .
$$

In practice we compute the product of two ratios:

$$
\frac{M}{\bar{m}\left(1 / 2 L_{\max }\right)}=\left(\frac{\bar{m}\left(1 / 2 L_{\max }\right)}{\bar{m}\left(1 / 2^{-k+1} L_{\max }\right)}\right)^{-1} \frac{M}{\bar{m}\left(1 / 2^{-k+1} L_{\max }\right)} .
$$

The first ratio on the r.h.s. is known from eq. (5.12). The second ratio, which refers to a perturbative scale $\mu=1 / 2^{-k+1} L_{\max }$, is calculated from eq. (5.13) with the NLO perturbative values of $\beta(g)$ and $\tau(g)$.

Having described the method, we gather the relevant results (and that of ref. [2]) in Table 4. The errors have been computed as outlined in Appendix B of ref. 2]. The following comments are in place:

(i) The quoted results have been obtained from the best SSF fits of eqs. (5.6) and (5.8). Several other fits, such as those described in the previous subsection, have been tried out. In all cases the final result $M / \bar{m}\left(1 / 2 L_{\max }\right)$ fluctuated within the quoted error, which in turn only increased slightly with increasing number of fitted parameters.

(ii) Our improved result is compatible with that of ref. 2]. The error is now smaller, due to improved statistics for the raw data on $Z_{\mathrm{P}}$.

(iii) Compared to our unimproved result, the improved one has a smaller error. Recalling that statistics are roughly the same, this reflects a better systematic control of discretisation effects, such as stable quadratic extrapolations in $(a / L)$. 
(iv) The result of the combined case is identical to the improved one.

Our final result is

$$
\frac{M}{\bar{m}\left(1 / 2 L_{\max }\right)}=1.155(9) \text {. }
$$

The quoted error does not include the effect of the uncertainty in the determination of $L_{\max } / r_{0}$, reported in ref. 20]. Its contribution being roughly equal to the above error implies that there is no point in increasing the precision of our result unless the uncertainty in $L_{\max } / r_{0}$ is also reduced.

\section{Conclusions}

We have performed a very detailed computation of the step scaling function of the quark mass in quenched lattice QCD, employing two variants of the lattice regularisation, namely unimproved and Clover-improved Wilson actions. In both cases the SSF has been computed at many renormalised gauge couplings (corresponding to a wide range of renormalisation scales) and for several lattice resolutions. Upon extrapolation to the continuum, the SSF has turned out to be independent of the specifics of the lattice regularisation, providing convincing evidence for the universality of the continuum limit.

The uniqueness of the continuum SSF has subsequently been used as a constraint, giving us an extra handle for the control of the sensitive extrapolations to zero lattice spacing. The final outcome of this detailed analysis, applied to high statistics data, is a very precise value of the ratio $M / \bar{m}(\mu)$. Far from being an academic exercise, the increased accuracy of our result, compared to [2, is of practical relevance. For example, in the context of the non-perturbative matching of Heavy Quark Effective Theory and QCD in finite volume, recently proposed and applied in refs. 21], precise numerical knowledge of the functional dependence of QCD observables on the renormalisation group invariant quark mass is of great importance.

The analysis described in this work is currently being applied to the SSF of other phenomenologically interesting quantities. Preliminary results on the SSF of the tensor bilinear operator $\bar{\psi} \sigma_{0 k} \psi$ (relevant e.g. to some semileptonic $B$-meson decays) have appeared in ref. [22, while the first results on the SSF of four-fermion operators (related to neutral meson oscillations, Kaon decays etc.) can be found in ref. [23].

\section{Acknowledgments}

We thank P. Hasenfratz, F. Niedermayer and S. Sint for discussions. Special thanks go to R. Sommer and H. Wittig for a critical reading of the manuscript and many 
helpful suggestions. C.P. acknowledges the financial support provided through the European Community's Human Potential Programme under contract HPRN-CT2000-00145, Hadrons/Lattice QCD. A.V. thanks the Bern Theory Group for its hospitality during the initial stages of this work. 

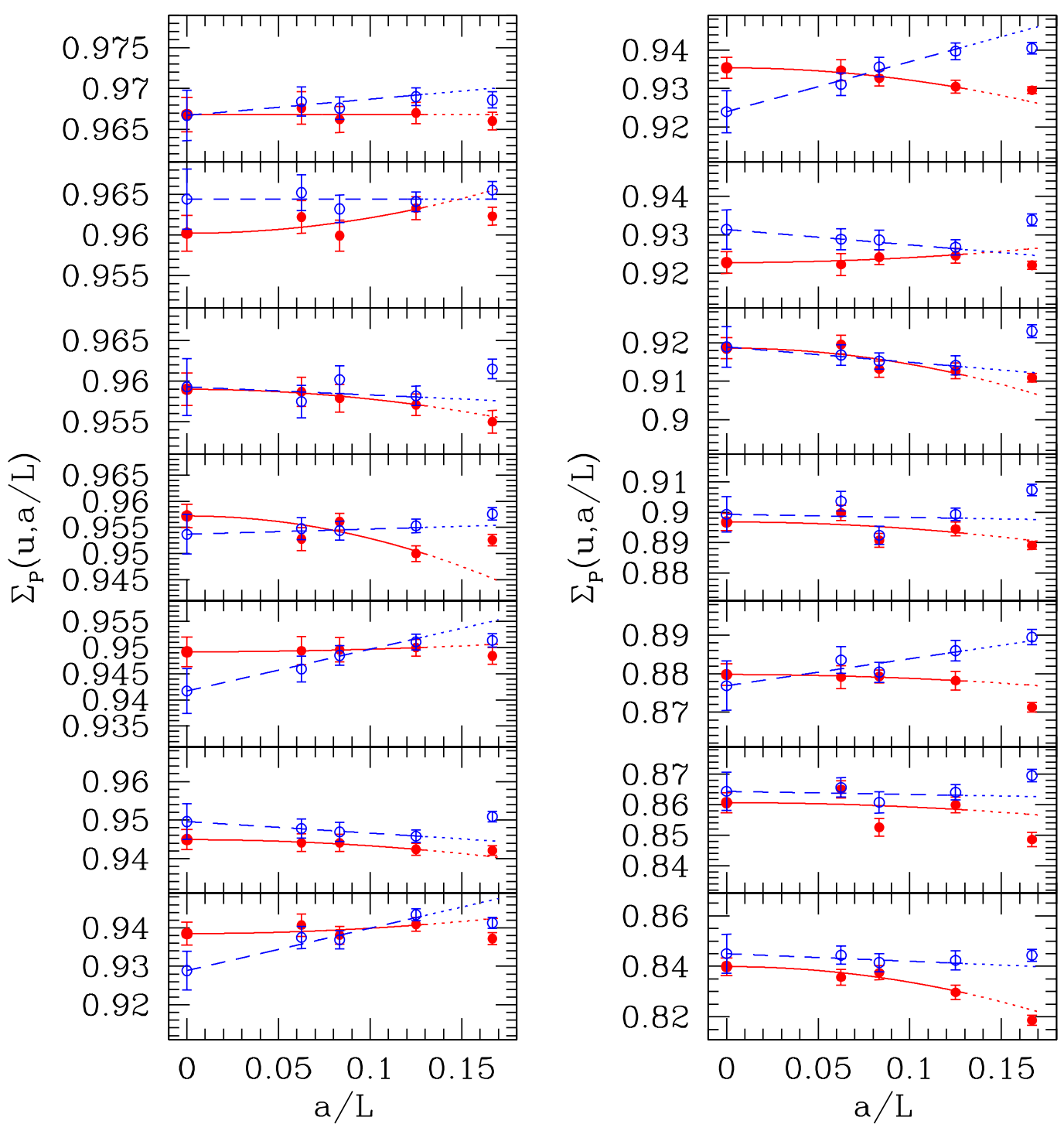

Figure 2: Continuum extrapolations of $\Sigma_{\mathrm{P}}$ at fixed renormalised coupling $u$ for the improved action (full symbols, solid line) and the unimproved action (open symbols, dashed line). The $L / a=6$ data points have not been included in the fits. The value of $u$ increases from top to bottom and from left to right. 

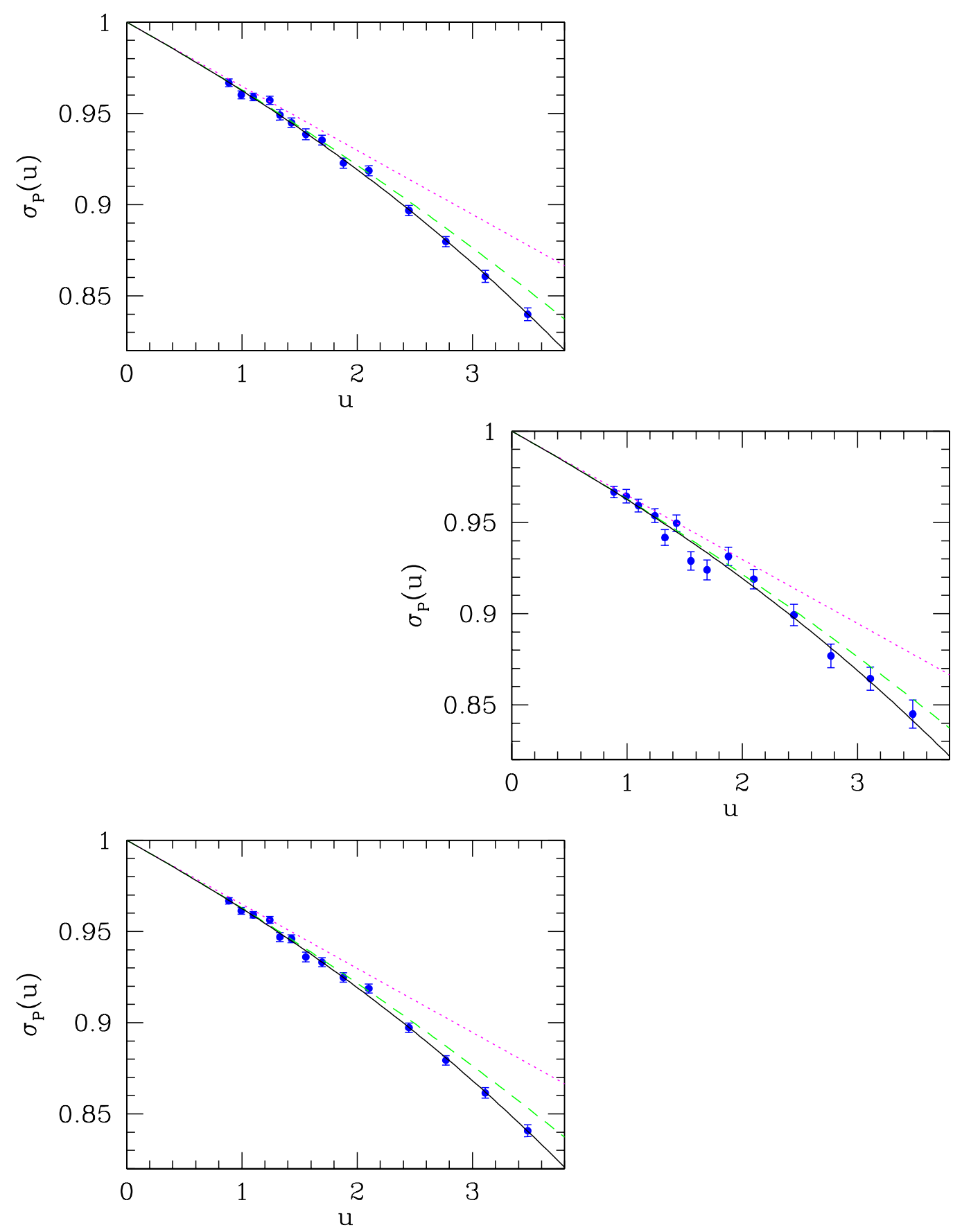

Figure 3: The step scaling function $\sigma_{\mathrm{P}}(u)$ (full points) in the improved (top), unimproved (middle) and combined (bottom) cases. Shown are also the expressions for the step scaling function in LO (dotted line) and NLO (dashed line) perturbation theory, as well as our best fit to the numerical data (solid line). 


\section{References}

[1] M. Lüscher, R. Sommer, P. Weisz and U. Wolff, Nucl. Phys. B413 (1994) 481.

[2] Alpha Collab., S. Capitani et al., Nucl. Phys. B544 (1999) 669.

[3] Alpha Collab., A. Bode et al., Phys. Lett. B 515 (2001) 49;

Alpha Collab., F. Knechtli et al., Nucl. Phys. Proc. Suppl. 119 (2003) 320;

Alpha Collab., M. Della Morte et al., Nucl. Phys. Proc. Suppl. 119 (2003) 439;

Alpha Collab., F. Knechtli et al., contribution by F. Knechtli at LATTICE 2003 (Tsukuba, July 2003); preprint HU-EP-03-61, DESY-03-137, SFB-CPP-03-38 and hep-lat/0309074.

[4] T. Reisz, Nucl. Phys. B318 (1989) 417.

[5] P. Hasenfratz and F. Niedermayer, Nucl. Phys. B596 (2001) 481;

M. Hasenbusch et al., Nucl. Phys. (Proc. Suppl.) 106 (2002) 911.

[6] Alpha Collab., G. de Divitiis et al., Nucl. Phys. B437 (1995) 447.

[7] S. Necco, Nucl. Phys. (Proc. Suppl) 119 (2003) 452; Nucl. Phys. B683 (2004) 137.

[8] M. Guagnelli, K. Jansen and R. Petronzio, Phys. Lett. B457 (1999) 153;

ZeRo Collab., M. Guagnelli et al., Nucl. Phys. B 664 (2003) 276.

[9] G.C. Rossi and M. Testa, Nucl. Phys. B163 (1980) 109; ibid. B176 (1980) 477;

ibid. B237 (1984) 442;

K. Symanzik, Nucl. Phys. B190[FS3] (1981) 1;

M. Lüscher, Nucl. Phys. B254 (1985) 52;

G.C. Rossi and K. Yoshida, Nuovo Cim. 11D (1989) 101;

J.P. Leroy, J. Micheli, G.C. Rossi and K. Yoshida, Z. Phys. C48 (1990) 653.

[10] M. Lüscher, R. Narayanan, P. Weisz and U. Wolff, Nucl. Phys. B384 (1992) 168.

[11] S. Sint, Nucl. Phys. B421 (1994) 135; ibid. B451 (1995) 416.

[12] M. Lüscher, S. Sint, R. Sommer and P. Weisz, Nucl. Phys. B478 (1996) 365.

[13] M. Lüscher et al., Nucl. Phys. B491 (1997) 323;

R.G. Edwards, U.M. Heller and T.R. Klassen, Nucl. Phys. B (Proc. Suppl.) 63 (1998) 847.

[14] R. Wohlert, "Improved continuum limit lattice action for quarks", DESY preprint 87-069 (1987), unpublished. 
[15] M. Lüscher and P. Weisz, Nucl. Phys. B479 (1996) 429.

[16] Alpha Collab., A. Bode, P. Weisz and U. Wolff, Nucl. Phys. B540 (1999) 491.

[17] K. Symanzik in: G 't Hooft t al., (eds.), "Recent developments in Gauge Theories" (Cargèse 1979) (Plenum, New York, 1980);

K. Symanzik in: R. Schrader et al., (eds.) "Mathematical Problems in Theoretical Physics", Lecture Notes in Physics, Vol. 153 (Springer, New York, 1982); K. Symanzik, Nucl. Phys. B226 (1983) 187; ibid., 205.

[18] Alpha Collab., S. Sint and P. Weisz, Nucl. Phys. B545 (1999) 529.

[19] Alpha Collab., M. Guagnelli, R. Sommer and H. Wittig, Nucl. Phys. B535 (1998) 389.

[20] S. Necco and R. Sommer, Nucl. Phys. B622 (2002) 328.

[21] Alpha Collab., J. Heitger and R. Sommer, JHEP 0402 (2004) 022;

Alpha Collab., J. Heitger and J. Wennekers, JHEP 0402 (2004) 064.

[22] Alpha Collab., M. Guagnelli, J. Heitger, C. Pena and A. Vladikas, contribution by C. Pena at LATTICE 2003 (Tsukuba, July 2003); preprint ROM2F-2003-21, MS-TP-03-11, DESY-03-130 and hep-lat/0309132.

[23] Alpha Collab., M. Guagnelli, J. Heitger, C. Pena, S. Sint and A. Vladikas, Nucl. Phys. (Proc. Suppl.) 119 (2003) 436. 